\title{
Registo Oncológico Animal
}

2020

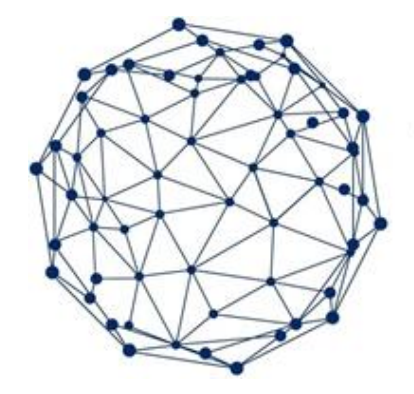

\section{Vet-OncoNet}

Veterinary Oncology Network

\section{ISPUP




\section{Contributos}

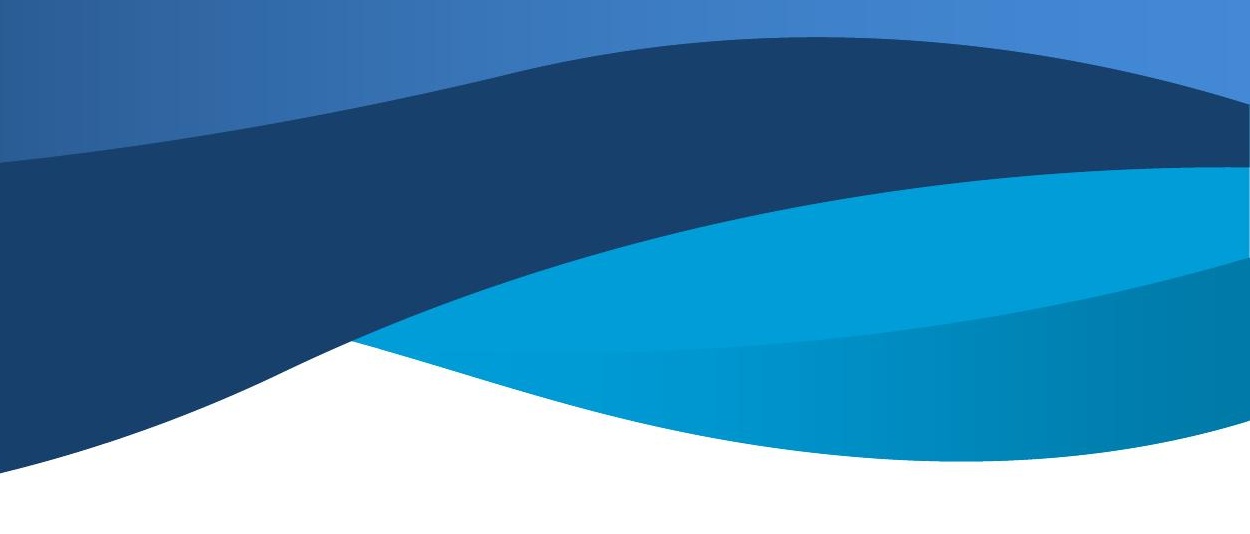

Grupo de Coordenação Vet-OncoNet

Prof. Doutor João Niza Ribeiro

Prof. Doutor Augusto de Matos

Prof. Doutora Katia Pinello

Prof. Doutora Andreia Santos

Prof. Doutora Felisbina Queiroga

Prof. Doutora Isabel Pires

\section{Responsável pela Gestão dos Dados}

Prof. Doutora Katia Pinello

\section{Produção do relatório}

Prof. Doutora Katia Pinello, Prof. Doutora Isabel Pires e Prof. Doutor João Niza

Ribeiro.

Patologistas Veterinários que contribuíram para a presente Edição (ordem alfabética)

Prof. Doutora Conceição Peleteiro

Prof. Doutora Irina Amorim

Prof. Doutor Pedro Faísca

Mestre José Catarino

\section{Contacto}

Vet-OncoNet

Rua de Jorge Viterbo Ferreira, 228

4050-313 Porto - Portugal

vetonconet@icbas.up.pt

\section{Edição}

Fevereiro 2021

\section{Produção}

Vet-OncoNet - Veterinary Oncology Network

Citação da publicação

Vet-OncoNet. Registo Oncológico Animal, 2020, Vol.1, $1^{\text {a }}$ Edição. 


\section{INTRODUÇÃO}

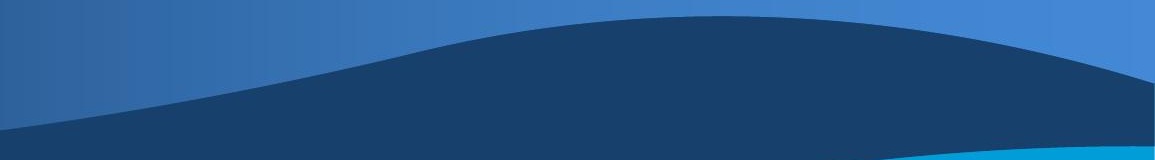

\section{.}

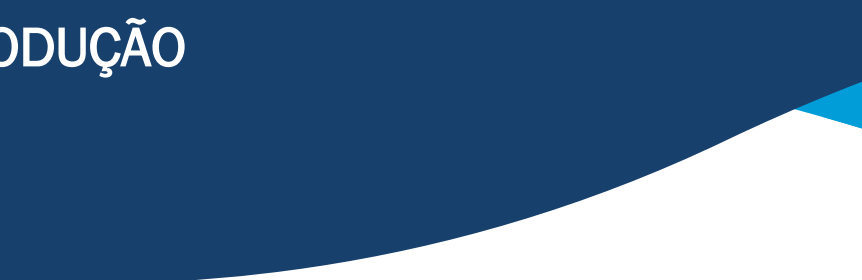

A presente publicação constitui o $1^{\circ}$ volume do Registo Oncológico Animal e contém informação sobre os casos de cancro em 2019 e 2020 registados pelos Laboratórios de diagnóstico veterinário aderentes da Rede Vet-OncoNet.

Os dados quando chegam à rede Vet-OncoNet passam por um processo de validação, uniformização e classificação. Foram registados neste relatório 8486 tumores, $80 \%$ da espécie canina, e 18,9\% da espécie felina. Destes, 2073 relativos a 2019 e 6413 a 2020.

A classificação de topografia e morfologia dos tumores baseou-se na estrutura da $3^{a}$ edição da International Classification of Diseases for Oncology (ICD-0-3.2) da medicina humana adaptada à medicina veterinária. Para permitir estabelecer algum tipo de comparação com os registos da medicina humana, os dados topográficos foram agrupados por aparelhos e sistemas conforme classificação utilizada pelo Registo Oncológico Regional do Norte (RORENO) 2012, edição de fevereiro de 2019 (Grupos topográficos).

As topografias mais frequentes foram pele, mama e tecidos moles e subcutâneo, que em conjunto representam quase $80 \%$ do total de casos. Em relação à morfologia, os principais tipos histológicos são lipomas, mastocitomas, carcinomas de células escamosas, adenoma complexo e histiocitomas.

As neoplasias mais frequentes foram registadas na espécie canina: mastocitomas $(9,4 \%)$, lipomas $(6,1 \%)$ e adenoma complexo da mama (4,7\%). Na espécie felina os mais frequentes são os carcinomas de células escamosas (12\%), adenocarcinoma tubular $(10,2 \%)$ e túbulo-papilar (7,8\%) da mama.

Quanto à distribuição por idade, praticamente metade dos tumores $(47,4 \%)$ ocorrem na faixa etária dos 8 aos 11 anos de idade, 49,4\% nos cães e 38,9\% nos gatos. 0 pico da incidência ocorre aos 10 anos de idade (12,8\%, 931/7276 casos).

Não estando disponíveis dados relativos ao censo canino e felino, não estão ainda reportados neste documento análises de frequência relativa.

Nota: sempre que é referida a espécie canina referimo-nos à subespécie Canis lupus familiaris e felina referimo-nos à espécie Felis catus.

A rede Vet-OncoNet agradece a todos os que contribuíram para este trabalho, nomeadamente à Direção do ICBAS, em especial ao Prof. Doutor Henrique Cyrne de Carvalho, e aos laboratórios de diagnóstico que acreditaram na importância de se criar um registo oncológico animal em Portugal. 


\section{ÍNDICE}

Casos registados no total por Espécie.

Distribuição dos tumores por Espécie e Sexo. 5

Distribuição dos casos por idade por espécie (canina e felina) 6

Distribuição geográfica dos casos por espécie (canina e felina) 6

Frequências dos tumores por aparelhos/sistemas por espécie (canina e felina)* 7

As Topografias mais frequentes por espécie (canina e felina) 7

As neoplasias (por morfologia) mais frequentes por espécie (canina e felina). 8

Distribuição dos tipos histológicos* por Grupos topográficos por espécie (canina e felina)...... 8

Lista de laboratórios de diagnóstico veterinário que enviaram dados à rede Vet-OncoNet em 2020. 10 


\section{CASOS REGISTADOS NO TOTAL POR ESPÉCIE}

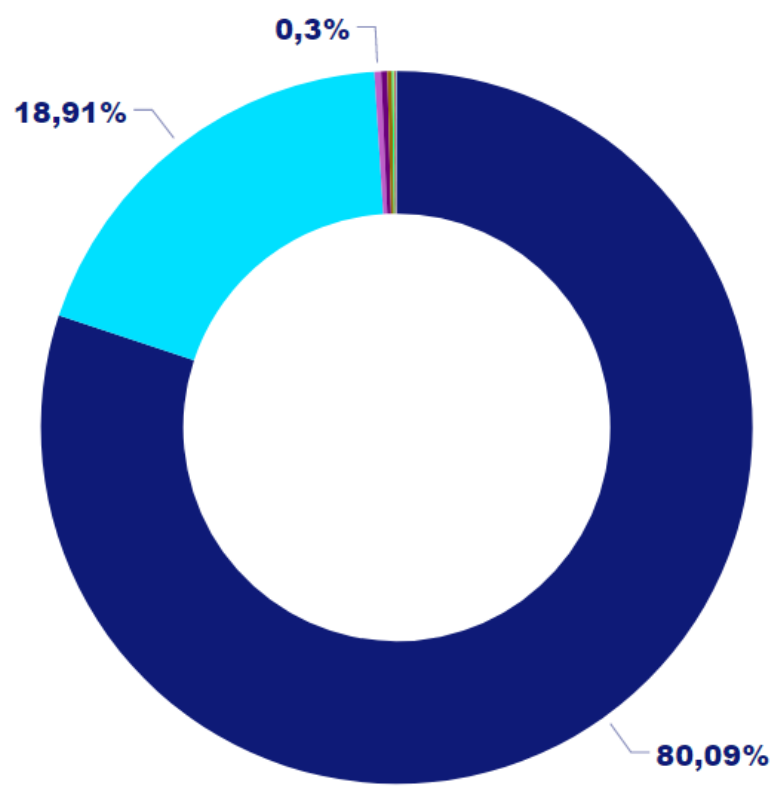

- Canina

- Felina

- Roedor

- Lagomorfo

- Equídeos

- Réptil

- Ave

- Furão

- Ouriço

- Bovino

- Leporídeo

- Peixe

- Pinguim

\section{DISTRIBUIÇÃO DOS TUMORES POR ESPÉCIE E SEXO}

\begin{tabular}{lcccccc} 
Espécie & Fêmea & $\mathbf{\%}$ & Macho & \% & Total & Acumulado \% \\
\hline Canina & 3931 & 58,4 & 2799 & 41,6 & 6730 & 80,5 \\
Felina & 1057 & 66,9 & 523 & 33,1 & 1580 & 99,0 \\
Lagomorfo & 18 & 75,0 & 6 & 25,0 & 24 & 99,2 \\
Roedores & 12 & 66,7 & 6 & 52,9 & 18 & 99,4 \\
Equídeos & 6 & 33,3 & 11 & 66,7 & 17 & 99.6 \\
Réptil & 1 & 16,7 & 3 & 83,3 & 6 & 99,7 \\
Furão & 1 & 33,3 & 2 & 66,7 & 3 & 99,8 \\
Pinguim & 1 & 100 & - & - & 1 & 99,8 \\
Ave & - & - & 1 & 100 & 1 & 99,9 \\
Peixe & - & - & 1 & 100 & 1 & 99,9 \\
Total Geral & $\mathbf{5 0 2 9}$ & $\mathbf{5 9 , 9}$ & $\mathbf{3 3 5 5}$ & $\mathbf{4 0 , 0}$ & $\mathbf{8 3 8 4}$ & $\mathbf{1 0 0}$ \\
\hline
\end{tabular}


DISTRIBUIÇÃO DOS CASOS POR IDADE POR ESPÉCIE (CANINA E FELINA)

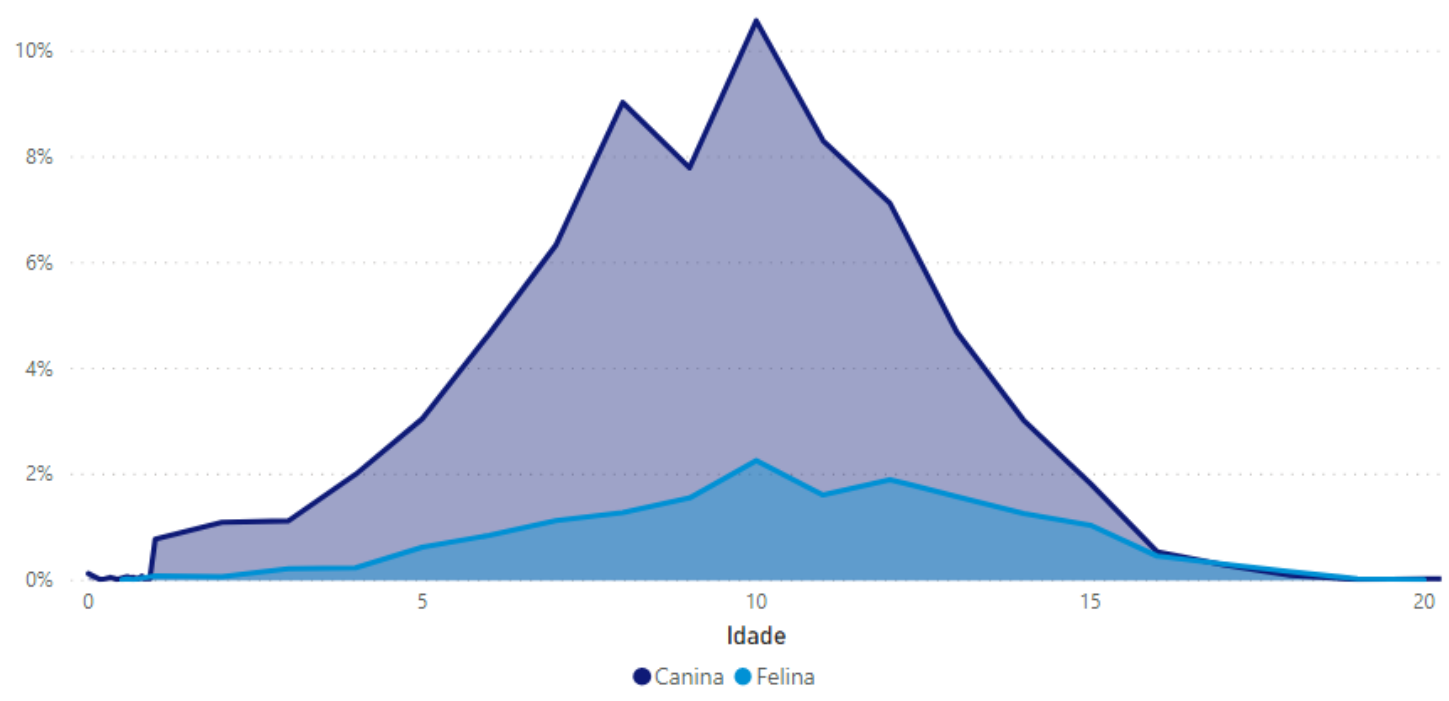

DISTRIBUIÇÃO GEOGRÁFICA DOS CASOS POR ESPÉCIE (CANINA E FELINA)

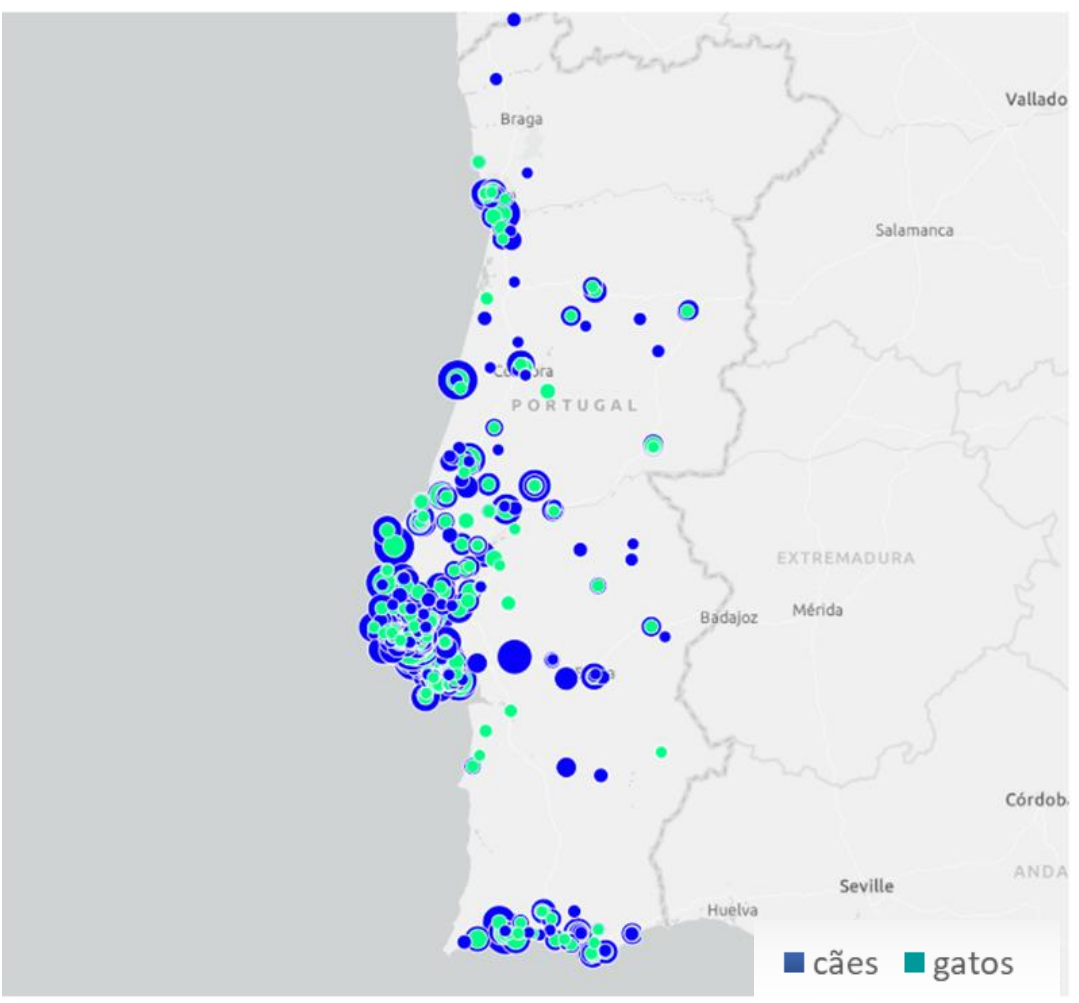


FREQUÊNCIAS DOS TUMORES POR APARELHOS/SISTEMAS POR ESPÉCIE (CANINA E FELINA)*

\begin{tabular}{|c|c|c|c|c|c|c|}
\hline \multirow{2}{*}{$\begin{array}{l}\text { Espécie } \\
\text { Grupos topográficos }\end{array}$} & \multicolumn{2}{|c|}{ Canina } & \multicolumn{2}{|l|}{ Felina } & \multicolumn{2}{|l|}{ Total } \\
\hline & $\mathrm{n}$ & $\%$ & $\mathrm{n}$ & $\%$ & $\mathrm{n}$ & $\%$ \\
\hline Pele & 3403 & $49,48 \%$ & 604 & $37,19 \%$ & 4007 & $47,14 \%$ \\
\hline Mama & 1558 & $22,66 \%$ & 592 & $36,45 \%$ & 2150 & $25,29 \%$ \\
\hline Órgãos Genito-Urinários & 513 & $7,46 \%$ & 23 & $1,42 \%$ & 536 & $6,31 \%$ \\
\hline Tecidos Moles & 439 & $6,38 \%$ & 46 & $2,83 \%$ & 485 & $5,71 \%$ \\
\hline Cavidade Oral e Faringe & 396 & $5,76 \%$ & 65 & $4,00 \%$ & 461 & $5,42 \%$ \\
\hline Peritoneu e Órgãos Digestivos & 105 & $1,53 \%$ & 131 & $8,07 \%$ & 236 & $2,78 \%$ \\
\hline Linfonodos & 93 & $1,35 \%$ & 45 & $2,77 \%$ & 138 & $1,62 \%$ \\
\hline Sistema Hematopoiético e Retículo Endotelial & 121 & $1,76 \%$ & 12 & $0,74 \%$ & 133 & $1,56 \%$ \\
\hline Sistema Respiratório e Órgãos Intra-Torácicos & 58 & $0,84 \%$ & 43 & $2,65 \%$ & 101 & $1,19 \%$ \\
\hline Primário de Origem Desconhecida & 71 & $1,03 \%$ & 14 & $0,86 \%$ & 85 & $1,00 \%$ \\
\hline Ossos e Articulações & 42 & $0,61 \%$ & 17 & $1,05 \%$ & 59 & $0,69 \%$ \\
\hline Olho e Glândula Lacrimal & 18 & $0,26 \%$ & 21 & $1,29 \%$ & 39 & $0,46 \%$ \\
\hline Glândulas Endócrinas & 26 & $0,38 \%$ & 6 & $0,37 \%$ & 32 & $0,38 \%$ \\
\hline Inespecíficos & 3 & $0,04 \%$ & & & 3 & $0,04 \%$ \\
\hline Sistema Nervoso & 1 & $0,01 \%$ & & & 1 & $0,01 \%$ \\
\hline Total & 6877 & $100,00 \%$ & 1624 & $100,00 \%$ & 8501 & $100,00 \%$ \\
\hline
\end{tabular}

* Grupos topográficos utilizados pelo RORENO

\section{AS TOPOGRAFIAS MAIS FREQUENTES POR ESPÉCIE (CANINA E FELINA)}

(1) Pele $50,9 \%$

(2) Mama 21,9\%

(3) Subcutâneo e tecidos moles $7,3 \%$

(4) Testículos 4,2\%

(5) Gengiva 3,3\%

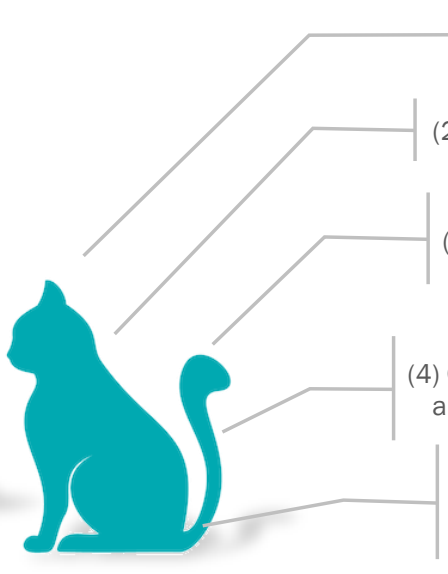

(1) Pele $38.7 \%$

(2) Mama 35,5\%

(3) Órgãos digestivos 6,1\%

4) Cavidade nasal e pavilhão auricular 3,1\%

(5) Subcutâneo e tecidos moles 3,0\% 


\section{AS NEOPLASIAS (POR MORFOLOGIA) MAIS FREQUENTES POR ESPÉCIE (CANINA E} FELINA)

(5) Tumor misto benigno da mama 3,7\%

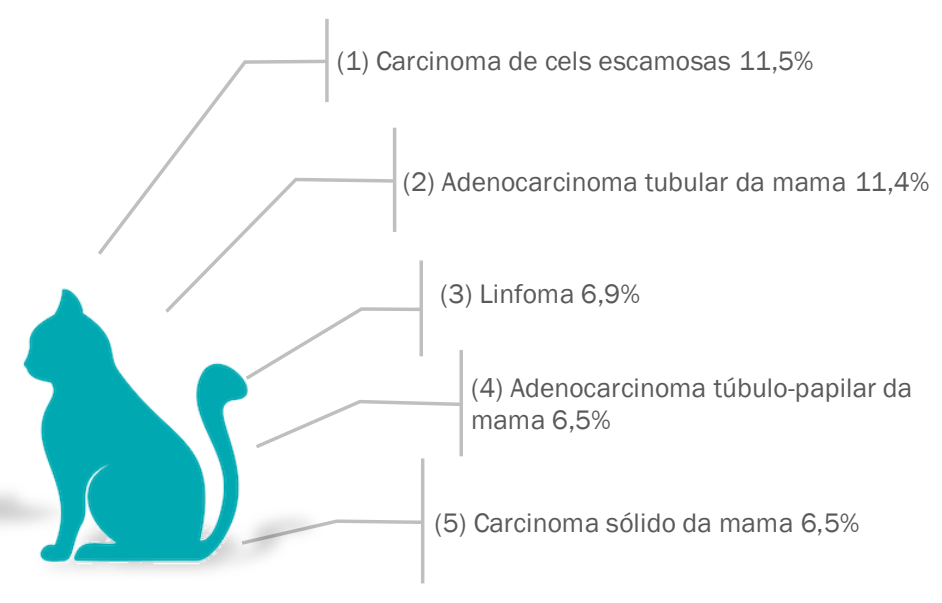

(Cães n=6877; Gatos n=1624)

\section{DISTRIBUIÇÃO DOS TIPOS HISTOLÓGICOS* POR GRUPOS TOPOGRÁFICOS POR ESPÉCIE \\ (CANINA E FELINA) *as 3 principais morfologias de cada grupo}

\begin{tabular}{|c|c|c|c|c|c|}
\hline \multirow{2}{*}{$\begin{array}{l}\text { Topografia } \\
\text { Morfologia }\end{array}$} & \multicolumn{2}{|c|}{ Canina } & \multirow{2}{*}{$\begin{array}{l}\text { Topografia } \\
\text { Morfologia }\end{array}$} & \multicolumn{2}{|c|}{ Felina } \\
\hline & $\mathbf{n}$ & $\% *$ & & $\mathbf{n}$ & $\% *$ \\
\hline Pele & 2931 & 50.0 & Pele & 520 & 38,6 \\
\hline $\begin{array}{l}\text { Mastocitoma } \\
\text { cutâneo baixo grau } \\
\text { Kiupel, grau II } \\
\text { Patnaik }\end{array}$ & 283 & 4,8 & $\begin{array}{l}\text { Carcinoma de } \\
\text { células escamosas }\end{array}$ & 133 & 9,8 \\
\hline Lipoma & 276 & 4.7 & Adenoma apócrino & 55 & 4,1 \\
\hline Histiocitoma & 222 & 3,8 & Fibrossarcoma & 45 & 3,3 \\
\hline Glândula mamária & 1283 & 21,9 & Glândula mamária & 478 & 35,5 \\
\hline Adenoma complexo & 274 & 4,7 & $\begin{array}{l}\text { Adenocarcinoma } \\
\text { tubular }\end{array}$ & 132 & 9.8 \\
\hline $\begin{array}{l}\text { Tumor misto } \\
\text { benigno }\end{array}$ & 223 & 3,8 & $\begin{array}{l}\text { Adenocarcinoma } \\
\text { túbulo-papilar }\end{array}$ & 105 & 7,8 \\
\hline Adenoma simples & 121 & 2,1 & Carcinoma sólido & 82 & 6.1 \\
\hline $\begin{array}{l}\text { Subcutâneo e tecidos } \\
\text { moles }\end{array}$ & 425 & 7,2 & $\begin{array}{l}\text { Subcutâneo e tecidos } \\
\text { moles }\end{array}$ & 40 & 2,9 \\
\hline Tumor perivascular & 192 & 3,3 & $\begin{array}{l}\text { Tumor de partes } \\
\text { moles, benigno }\end{array}$ & 10 & 0,74 \\
\hline $\begin{array}{l}\text { Tumor de partes } \\
\text { moles, benigno }\end{array}$ & 77 & 1,32 & Fibrossarcoma & 10 & 0,74 \\
\hline $\begin{array}{l}\text { Mastocitomas } \\
\text { subcutâneo, } \\
\text { padrão infiltrativo }\end{array}$ & 41 & 0,7 & Sarcoma histiocítico & 5 & 0,37 \\
\hline
\end{tabular}




\begin{tabular}{|c|c|c|c|c|c|}
\hline $\begin{array}{l}\text { Órgãos Génito- } \\
\text { urinários }\end{array}$ & 414 & 7,07 & $\begin{array}{l}\text { Órgãos Génito- } \\
\text { urinários }\end{array}$ & 22 & 1,63 \\
\hline $\begin{array}{l}\text { Tumor das céls de } \\
\text { Leydig }\end{array}$ & 66 & 1,13 & $\begin{array}{l}\text { Adenocarcinoma, } \\
\text { SOE }\end{array}$ & 8 & 0,59 \\
\hline $\begin{array}{l}\text { Tumor das céls de } \\
\text { Sertoli }\end{array}$ & 59 & 1,0 & Carcinoma urotelial & 3 & 0,22 \\
\hline Seminoma & 50 & 0,85 & Leiomioma & 3 & 0,22 \\
\hline $\begin{array}{l}\text { Cavidade Oral e } \\
\text { Faringe }\end{array}$ & 322 & 5,50 & $\begin{array}{l}\text { Cavidade Oral e } \\
\text { Faringe }\end{array}$ & 52 & 3,86 \\
\hline $\begin{array}{l}\text { Fibroma } \\
\text { odontogénico } \\
\text { periférico }\end{array}$ & 97 & 1,6 & $\begin{array}{l}\text { Carcinoma de céls } \\
\text { escamosas }\end{array}$ & 22 & 1,63 \\
\hline $\begin{array}{l}\text { Ameloblastoma } \\
\text { acantomatoso }\end{array}$ & 32 & 0,55 & Fibrossarcoma & 9 & 0,67 \\
\hline Melanoma & 26 & 0,44 & $\begin{array}{l}\text { Fibroma } \\
\text { odontogénico }\end{array}$ & 3 & 0,22 \\
\hline $\begin{array}{l}\text { Peritoneu e Órgãos } \\
\text { digestivos }\end{array}$ & 87 & 1,49 & $\begin{array}{l}\text { Peritoneu e Órgãos } \\
\text { digestivos }\end{array}$ & 107 & 7,95 \\
\hline $\begin{array}{l}\text { Adenoma } \\
\text { hepatocelular }\end{array}$ & 12 & 0,21 & Linfoma & 68 & 5,05 \\
\hline Leiomiossarcoma & 12 & 0,21 & $\begin{array}{l}\text { Adenocarcinoma } \\
\text { intestinal }\end{array}$ & 7 & 0,52 \\
\hline $\begin{array}{l}\text { Adenocarcinoma } \\
\text { intestinal }\end{array}$ & 9 & 0,15 & Linfoma T intestinal & 6 & 0,45 \\
\hline Sistema & 105 & 1,79 & Sistema & 9 & 0,67 \\
\hline $\begin{array}{l}\text { Hematopoiético e } \\
\text { Reticuloendotelial }\end{array}$ & & & $\begin{array}{l}\text { Hematopoiético e } \\
\text { Reticuloendotelial }\end{array}$ & & \\
\hline Hemangiosarcoma & 54 & 0,92 & Hemangiosarcoma & 3 & 0,22 \\
\hline Linfoma, SOE & 13 & 0,22 & $\begin{array}{l}\text { Linfoma hépato- } \\
\text { esplénico }\end{array}$ & 2 & 0,15 \\
\hline Mielolipoma & 9 & 0,15 & Mesotelioma & 1 & 0,07 \\
\hline $\begin{array}{l}\text { Sistema respiratório e } \\
\text { Órgãos Intratorácicos }\end{array}$ & 56 & 0,56 & $\begin{array}{l}\text { Sistema respiratório e } \\
\text { Órgãos Intratorácicos }\end{array}$ & 42 & 3,12 \\
\hline $\begin{array}{l}\text { Adenocarcinoma, } \\
\text { SOE }\end{array}$ & 5 & 0,09 & Fibrossarcoma & 7 & 0,52 \\
\hline Histiocitoma & 5 & 0,09 & $\begin{array}{l}\text { Adenocarcinoma, } \\
\text { SOE }\end{array}$ & 5 & 0,37 \\
\hline $\begin{array}{l}\text { Adenoma } \\
\text { ceruminoso }\end{array}$ & 3 & 0,05 & $\begin{array}{l}\text { Adenoma } \\
\text { ceruminoso }\end{array}$ & 5 & 0,37 \\
\hline Linfonodos & 70 & 1,20 & Linfonodos & 25 & 1,86 \\
\hline Linfoma, SOE & 54 & 0,92 & Linfoma, SOE & 14 & 1,04 \\
\hline Metástases & 10 & 0,17 & Metástases & 4 & 0,30 \\
\hline $\begin{array}{l}\text { Linfoma de Zona T, } \\
\text { nodal }\end{array}$ & 2 & 0,03 & $\begin{array}{l}\text { Metástase de } \\
\text { carcinoma }\end{array}$ & 4 & 0,30 \\
\hline Ossos e articulações & 41 & 0,70 & Ossos e articulações & 14 & 1,04 \\
\hline Osteossarcoma & 26 & 0,44 & Osteossarcoma & 3 & 0,22 \\
\hline $\begin{array}{l}\text { Osteossarcoma } \\
\text { condroblástico }\end{array}$ & 2 & 0,03 & $\begin{array}{l}\text { Osteossarcoma } \\
\text { condroblástico }\end{array}$ & 2 & 0,15 \\
\hline
\end{tabular}


Osteossarcoma 2

fibroblástico

Olho

Melanocitoma

Melanoma

Glândulas endócrinas

Carcinoma

folicular, SOE

Adenoma cortical

da adrenal

Adenoma folicular,

SOE

$\begin{array}{lcc}\begin{array}{l}\text { Sistema Nervoso } \\ \text { Schwanoma, SOE }\end{array} & \mathbf{1} & \mathbf{0 , 0 2} \\ \begin{array}{l}\text { Primário de origem } \\ \text { desconhecida } \\ \text { Lipoma }\end{array} & \mathbf{5 8} & \mathbf{0 , 9 9} \\ \quad \text { Tumor perivascular } & 4 & 0,26 \\ \quad \text { Mastocitoma } & 3 & 0,05 \\ \quad \text { subcutâneo, } \\ \text { padrão infiltrativo }\end{array}$

\begin{tabular}{l|lcc}
0,03 & $\begin{array}{l}\text { Osteossarcoma } \\
\text { fibroblástico }\end{array}$ & 2 & 0,15
\end{tabular}

\begin{tabular}{cc|lcc}
15 & $\mathbf{0 , 2 6}$ & Olho & $\mathbf{1 6}$ & $\mathbf{1 , 1 9}$ \\
4 & 0,07 & Melanoma & 7 & 0,52 \\
3 & 0,05 & Sarcoma & 2 & 0,15
\end{tabular}

\begin{tabular}{cc|lll}
17 & 0,29 & $\begin{array}{l}\text { Glândulas endócrinas } \\
\text { Adenoma folicular, }\end{array}$ & 6 & 0,45 \\
5 & 0,09 & $\begin{array}{l}\text { SOE } \\
\text { Adenoma cortical da } \\
\text { adrenal }\end{array}$ & 2 & 0,15 \\
\hline & 0,05 & &
\end{tabular}

* Percentagens do total de neoplasias por espécie. SOE: Sem Outra Especificação.

\title{
LISTA DE LABORATÓRIOS DE DIAGNÓSTICO VETERINÁRIO QUE ENVIARAM DADOS À REDE VET-ONCONET EM 2020.
}

\author{
Instituição \\ DNATech \\ VetPatLab Análises Anatomopatológicas Veterinárias Lda \\ Laboratório de Anatomia Patológica da Faculdade de Medicina Veterinária de Lisboa
}

